\title{
Fuzzy Lattice Ordered M-Group
}

\author{
M.U. Makandar \\ Assistant Professor. PG, \\ KIT's IMER. \\ Shivaji University, Kolhapur.
}

\author{
A.D. Lokhande, Ph.D \\ H.O.D. Dept of Mathematics. \\ Y.C.Warana Mahavidyalaya \\ Warananager
}

\begin{abstract}
In this paper we introduce the notion of fuzzy lattice ordered m-groups and investigated some of its basic properties. We also study the homomorphic image, preimage of fuzzy lattice ordered m-groups, arbitrary family of fuzzy lattice ordered m-groups and fuzzy lattice ordered m-groups using T-norms. We introduce the notion of sensible fuzzy lattice ordered m-groups in groups and some related properties of lattices are discussed.
\end{abstract}

\section{Keywords:}

Lattice ordered group, Fuzzy lattice ordered m-group, Sensible fuzzy lattice, pre-image, direct product.

\section{INTRODUCTION}

A fuzzy algebra has become an important branch of research. A. Rosenfeld 1971 [9] used the concept of fuzzy set theory due to Zadeh 1965 [5]. Since then the study of fuzzy algebraic substructures are important when viewed from a Lattice theoretic point of view. N. Ajmal and K.V. Thomas [1] initiated such types of study in the year 1994. It was latter independently established by $\mathrm{N}$. Ajmal [1] that the set of all fuzzy normal subgroups of a group constitute a sub lattice of the lattice of all fuzzy sub groups of a given group and is Modular. Nanda[8] proposed the notion of fuzzy lattice using the concept of fuzzy partial ordering. More recently in the notion of set product is discussed in details and in the lattice theoretical aspects of fuzzy sub groups and fuzzy normal sub groups are explored. G.S.V. Satya Saibaba [3] initiate the study of L-fuzzy lattice ordered groups and introducing the notice of L-fuzzy sub 1- groups. J.A. Goguen [4] replaced the valuation set $[0,1]$ by means of a complete lattice in an attempt to make a generalized study of fuzzy set theory by studying L-fuzzy sets. A Solairaju and R. Nagarajan [11] introduced the concept of lattice valued Q-fuzzy sub-modules over near rings with respect to T-norms. Dr M.Marudai \& V. Rajendran[6] modified the definition of fuzzy lattice and introduce the notion of fuzzy lattice of groups and investigated some of its basic properties. Gu [12] introduced concept of fuzzy groups with operator. Then S. Subramanian, R Nagrajan \& Chellappa [10] extended the concept to $m$ fuzzy groups with operator. In this paper we introduce the notion of fuzzy lattice ordered m-groups and investigated some of its basic properties. We study the homomorphic image, pre-image of fuzzy lattice ordered m-groups, arbitrary family of fuzzy lattice ordered m-groups and fuzzy lattice ordered $\mathrm{m}$ normal groups. We introduce the notion of sensible fuzzy lattice ordered $\mathrm{m}$-groups in groups using $\mathrm{T}$ norms and some related properties of lattices are discussed.. We introduce here the notion of fuzzy lattice ordered m-groups and investigated some of its basic properties.

\section{PRELIMINARIES}

Definition 2.1: Let $\mu: X \rightarrow[0,1]$ be a fuzzy set $\& \mathrm{G}$ $\epsilon p(X)=$ Set of all fuzzy sets on $X$. A fuzzy set $\mu$ on $G$ is called a fuzzy group if i) $\mu(\mathrm{x} y) \geq \min \{\mu(\mathrm{x}), \mu(\mathrm{y})\}$ ii) $\mu$ $\left(\mathrm{x}^{-1}\right) \geq \mu(\mathrm{x})$, for all $\mathrm{x}$, yє G .

Definition 2.2: Let $\mu: X \rightarrow[0,1]$ be a fuzzy set $\& G$ $\epsilon b(X)$. A fuzzy set $\mu$ on $G$ is called a normal fuzzy subgroup if $\mu\left(\mathrm{x}^{-1} \mathrm{y} \mathrm{x}\right) \geq \mu(\mathrm{y}) \quad$ for all $\mathrm{x}, \mathrm{y} \in \mathrm{G}$

Definition 2.3: A lattice ordered group is a system ( $\mathrm{G}, ., \leq)$ if $\mathrm{i})(\mathrm{G}, \cdot)$ is a group ii) $(\mathrm{G}, \leq)$ is a lattice . iii $) \mathrm{x} \leq \mathrm{y}$ implies a $\mathrm{x} \mathrm{b} \leq \mathrm{a}$ y $\mathrm{b}$ ( compatibility)

For a, b, x, yє G

Definition 2.4: Let $\mu: X \rightarrow[0,1]$ be a fuzzy set $\& G$ is a lattice ordered set, $G \in p(X)$. A function $\mu$ on $G$ is said to be a fuzzy lattice ordered group if i) $\mu$ (x y) $\geq \min \{\mu$ (x), $\mu(\mathrm{y})\}$ ii) $\mu\left(\mathrm{x}^{-1}\right) \geq \mu$ (x) for all $\mathrm{x}, \mathrm{y} \in \mathrm{G}$

Definition 2.5: Let $G$ be a group, $M$ be any set if $i$ ) $m x \in G$. ii) $m(x y)=(m x) y=x m y$ for all $x, y \in G, m \epsilon$ $M$. Then $\mathrm{G}$ is called a $\mathrm{m}$ group.

Definition 2.6: - Let $\mu: X \rightarrow[0,1]$ be a fuzzy set \& $\mathrm{G}$ be a $\mathrm{M}$ group $\mathrm{G}$. A fuzzy set on $\mathrm{G}, \mathrm{G} \epsilon \mathrm{p}(\mathrm{X})$ ) is called a fuzzy $\mathrm{m}$ group if i) $\mu(\mathrm{m}(\mathrm{x} y)) \geq \min \{\mu(\mathrm{m} \mathrm{x}), \mu$ (my) $\}$ ii) $\mu\left(\mathrm{mx}^{-1}\right) \geq \mu(\mathrm{mx}) \quad$ for all $\mathrm{x}, \mathrm{y} \in \mathrm{G}, \mathrm{m} \in \mathrm{M}$

Definition 2.7: A t-norm $\mathrm{T}$, we mean a function $\mathrm{T}$ : $[0,1] \times[0,1][0,1]$ satisfying the following conditions:

(T1) $\mathrm{T}(0, \mathrm{X})=0$

(T2) $\mathrm{T}(\mathrm{x}, \mathrm{y}) \mathrm{T}(\mathrm{x}, \mathrm{z})$ if $\mathrm{y} \mathrm{z}$

(T3) $\mathrm{T}(\mathrm{x}, \mathrm{y})=\mathrm{T}(\mathrm{y}, \mathrm{x})$

(T4) $\mathrm{T}(\mathrm{x}, \mathrm{T}(\mathrm{y}, \mathrm{z}))=\mathrm{T}(\mathrm{T}(\mathrm{x}, \mathrm{y}), \mathrm{z})$, for all $\mathrm{x}, \mathrm{y}, \mathrm{z} \in[0,1]$

Definition 2.8: Let $\mathrm{T}$ be a t-norm. A fuzzy set $\mathrm{A}$ is said to be sensible under $\mathrm{T}$ if $\operatorname{Im}(\mathrm{A}) \mathrm{C} \Delta \mathrm{T}$. Where $\Delta \mathrm{T}=\{\alpha \in[0,1] / \mathrm{T}(\alpha, \alpha)=\alpha\}$ 
Definition 2.9: For any fuzzy $m$ group $G$ and $t \epsilon[0$, 1], We define the set $U(\mu: t)=\{x \in G \mid \mu(m x) \geq t\}$ which is called an upper cut off $\mu$ and can be used to the characterization of $\mu$.

Definition 2.10: Let $\theta: X \rightarrow Y$ be a map. $A$ and $B$ are fuzzy lattice ordered $\mathrm{m}$ groups in $\mathrm{X}$ and $\mathrm{Y}$ respectively. Then the inverse image of $\mathrm{B}$ under $\Theta$ is a fuzzy set defined by

$$
\Theta^{-1}(B)=\mu_{\theta^{-1}(B)}(x)=\mu_{B} \theta(x)
$$

Definition 2.11: Let $\mu_{A}$ be a fuzzy set of G. Let $\theta$ : $\mathrm{G} \rightarrow \mathrm{G}$ 'be a map.

Define the map $\mu_{A^{\ominus}}: \mathrm{G} \rightarrow[0,1]$ by $\mu_{A^{\ominus}}(\mathrm{x})=\mu_{A} \Theta(\mathrm{x})$

Definition 2.12: Let $f: G \rightarrow G$ ' be a lattice group homomorphism and A be a fuzzy lattice of $\mathrm{G}^{\prime}$ then $\operatorname{Af}(\mathrm{x})$ $=(A \circ f)(x)=f^{-1}(A)(x)$.

Definition 2.13: Let $\mu$ : $X$ to $[0,1], G \in b(X), M C$ $\mathrm{X}$. A function $\mu$ on $\mathrm{G}$ is said to be a fuzzy lattice ordered $\mathrm{m}$-group if

i) $(\mathrm{G}, \cdot)$ is a $\mathrm{M}$-group.

ii) $(\mathrm{G}, \cdot, \leq)$ is a lattice ordered group.

iii) $\mu(\mathrm{m}(\mathrm{x} y)) \geq \min \{\mu(\mathrm{m} x), \mu(\mathrm{my})\}$

iv) $\mu\left((\mathrm{mx})^{-1}\right) \geq \mu(\mathrm{mx})$

v) $\mu(\mathrm{mx} \mathrm{v} \mathrm{my}) \geq \min \{\mu(\mathrm{mx}), \mu(\mathrm{m} \mathrm{y})\}$

vi) $\mu(\mathrm{mx} \wedge \mathrm{my}) \geq \min \{\mu(\mathrm{mx}), \mu(\mathrm{my})\}$

For all $\mathrm{x}$, $y \in G$

\section{PROPERTIES OF FUZZY LATTICE ORDERED M-GROUP}

Proposition 3.1: $\quad$ Let $G$ and G' be two fuzzy lattice ordered m-groups and $\theta: G \rightarrow G^{\prime}$ be a $m$ homomorphism defined by $\theta(\mathrm{mx})=\mathrm{m} \theta(\mathrm{x})$.If $\mathrm{B}$ is a fuzzy lattice ordered $m$-group of $G^{\prime}$ then the pre-image $\theta^{-}$ ${ }^{1}(B)$ is a fuzzy lattice ordered m-group of $\mathrm{G}$.

Proof- Assume B is a fuzzy lattice ordered m-group of G'. Let $x, y \in G$

i) $\mu_{\theta^{-1}(\mathrm{~B})}(\mathrm{m}(\mathrm{x} y))=\mu_{\mathrm{B}} \theta(\mathrm{m}(\mathrm{x} y))$

$=\mu_{\mathrm{B}}(\mathrm{m} \theta(\mathrm{x} \mathrm{y}))$

$=\mu_{\mathrm{B}}(\mathrm{m} \theta(\mathrm{x}) \Theta(\mathrm{y}))$

$\geq \min \left\{\mu_{\mathrm{B}}(\mathrm{m} \theta(\mathrm{x})), \mu_{\mathrm{B}}(\mathrm{m} \theta(\mathrm{y}))\right\}$

$\geq \min \left\{\mu_{\mathrm{B}}(\Theta(\mathrm{m} \mathrm{x})), \mu_{\mathrm{B}}(\Theta(\mathrm{m} \mathrm{y}))\right\}$

$\geq \min \left\{\mu_{\theta^{-1}(\mathrm{~B})}(\mathrm{m} \mathrm{x}), \mu_{\mathrm{e}^{-1}(\mathrm{~B})}(\mathrm{m} \mathrm{y})\right\}$

ii) $\left.\mu_{\mathrm{A}^{-1}(\mathrm{~B})}(\mathrm{m} \mathrm{x})^{-1}\right)=\mu_{\mathrm{B}} \mathrm{\theta}\left((\mathrm{mx})^{-1}\right)$

$=\mu_{\mathrm{B}}(\mathrm{\theta}(\mathrm{m} \mathrm{x}))^{-1}$

$=\mu_{\mathrm{B}}(\mathrm{m} \theta(\mathrm{x}))^{-1}$

$\geq \mu_{\mathrm{B}}(\mathrm{m} \theta(\mathrm{x}))$

$\geq \mu_{\mathrm{B}}(\mathrm{\theta}(\mathrm{m} \mathrm{x}))$

$\geq \mu_{\theta^{-1}(\mathrm{~B})}(\mathrm{m} \mathrm{x})$

iii) $\mu_{\mathrm{e}^{-1}(\mathrm{~B})}(\mathrm{m} \times \mathrm{x} \vee \mathrm{my})=\mu_{\mathrm{B}} \mathrm{\theta}(\mathrm{m} \mathrm{x} \vee \mathrm{m} \mathrm{y})$
$=\mu_{\mathrm{B}} \mathrm{\theta}(\mathrm{mx}) \mathrm{v} \Theta(\mathrm{my})$

$\geq \min \left\{\mu_{\mathrm{B}} \Theta(\mathrm{mx}), \mu_{\mathrm{B}} \Theta(\mathrm{m} \mathrm{y})\right)$

$\geq \min \left\{\mu_{\theta^{-1}(\mathrm{~B})}(\mathrm{m} \mathrm{x}), \mu_{\theta^{-1}(\mathrm{~B})}(\mathrm{m} \mathrm{y})\right\}$

iv) $\mu_{\theta^{-1}(\mathrm{~B})}(\mathrm{m} \times \Lambda \mathrm{my})=\mu_{\mathrm{B}} \theta(\mathrm{mx} \Lambda \mathrm{m} \mathrm{y})$

$=\mu_{\mathrm{B}} \mathrm{\theta}(\mathrm{mx}) \Lambda \mathrm{\theta}(\mathrm{my})$

$\geq \min \left\{\mu_{\mathrm{B}} \Theta(\mathrm{mx}), \mu_{\mathrm{B}} \Theta(\mathrm{m} \mathrm{y})\right)$

$\geq \min \left\{\mu_{\theta^{-1}(\mathrm{~B})}(\mathrm{m} \mathrm{x}), \mu_{\theta^{-1}(\mathrm{~B})}(\mathrm{m} \mathrm{y})\right\}$

Therefore $\theta^{-1}(B)$ is a fuzzy lattice ordered $m$-group of $G$.

Proposition 3.2: Let G and G' be two fuzzy lattice ordered m-groups and $\theta: G \rightarrow G$ ' be a m-epimorphism. $B$ is a fuzzy set in $G^{\prime}$. If $e^{-1}(B)$ is a fuzzy lattice ordered $m$ group of $\mathrm{G}$ then $\mathrm{B}$ is a fuzzy lattice ordered group of $\mathrm{G}^{\prime}$.

Proof- Let $x, y \in G$ ', therefore there exist an element a, $\mathrm{b} \in \mathrm{G}$ such that

$\theta(a)=x$ and

$\theta(\mathrm{b})=\mathrm{y}$.

i) $\mu_{\mathrm{B}}(\mathrm{m}(\mathrm{x} \mathrm{y})) \quad=\mu_{\mathrm{B}}(\mathrm{m}(\mathrm{\theta}(\mathrm{a}) \mathrm{\theta}(\mathrm{b}))$

$=\mu_{\mathrm{B}}(\mathrm{m} \Theta(\mathrm{a} \mathrm{b}))$

$=\mu_{\mathrm{B}} \Theta(\mathrm{m}(\mathrm{a} \mathrm{b}))$

$=\mu_{\mathrm{\theta}^{-1}(\mathrm{~B})}(\mathrm{m}(\mathrm{a} \mathrm{b}))$

$\left.\left.\geq \min \left\{\mu_{\theta^{-1}(\mathrm{~B})}(\mathrm{ma})\right), \mu_{\theta^{-1}(\mathrm{~B})}(\mathrm{m} \mathrm{b})\right)\right\}$

$\geq \min \left\{\mu_{\mathrm{B}} \Theta(\mathrm{m} \mathrm{a}), \mu_{\mathrm{B}} \Theta(\mathrm{m} \mathrm{b})\right\}$

$\geq \min \left\{\mu_{\mathrm{B}} \mathrm{m} \Theta(\mathrm{a}), \mu_{\mathrm{B}} \mathrm{m} \Theta(\mathrm{b})\right\}$

$\geq \min \left\{\mu_{\mathrm{B}}(\mathrm{mx}), \mu_{\mathrm{B}}\right.$ (my ) $\}$

ii) $\mu_{\mathrm{B}}\left((\mathrm{m} \mathrm{x})^{-1}\right) \quad=\mu_{\mathrm{B}}(\mathrm{m} \Theta(\mathrm{a}))^{-1}$

$=\mu_{\mathrm{B}}(\mathrm{\theta}(\mathrm{ma}))^{-1}$

$=\mu_{\mathrm{B}}\left(\Theta(\mathrm{m} \mathrm{a})^{-1}\right)$

$=\mu_{\theta^{-1}(\mathrm{~B})}(\mathrm{m} \mathrm{a})^{-1}$

$\geq \mu_{\theta^{-1}(\mathrm{~B})}(\mathrm{m} \mathrm{a})$

$\geq \mu_{\mathrm{B}} \theta(\mathrm{ma})$

$\geq \mu_{\mathrm{B}} \mathrm{m} \theta(\mathrm{a})$

$\geq \mu_{\mathrm{B}}(\mathrm{mx})$

iii) $\mu_{\mathrm{B}}(\mathrm{m} \mathrm{x} \vee \mathrm{m} \mathrm{y})=\mu_{\mathrm{B}}(\mathrm{m} \Theta(\mathrm{a}) \vee \mathrm{m} \Theta(\mathrm{b}))$

$=\mu_{\mathrm{B}}(\Theta(\mathrm{m} \mathrm{a}) \vee \mathrm{\theta}(\mathrm{m} \mathrm{b}))$

$=\mu_{\mathrm{B}}(\Theta(\mathrm{m} \mathrm{a} \mathrm{v} \mathrm{m} \mathrm{b}))$

$=\mu_{\mathrm{\theta}^{-1}(\mathrm{~B})}(\mathrm{m} \mathrm{a} \vee \mathrm{m} \mathrm{b})$

$\geq \min \left\{\mu_{\theta^{-1}(\mathrm{~B})}(\mathrm{ma}), \mu_{\theta^{-1}(\mathrm{~B})}(\mathrm{m} \mathrm{b})\right\}$

$\geq \min \left\{\mu_{\mathrm{B}} \theta(\mathrm{ma}), \mu_{\mathrm{B}} \theta(\mathrm{mb})\right)$

$\geq \min \left\{\mu_{\mathrm{B}} \mathrm{m} \theta(\mathrm{a}), \mu_{\mathrm{B}} \mathrm{m} \theta(\mathrm{b})\right)$

$\geq \min \left\{\mu_{\mathrm{B}}(\mathrm{m} \mathrm{x}), \mu_{\mathrm{B}}(\mathrm{m} \mathrm{y})\right\}$

iv) $\mu_{\mathrm{B}}(\mathrm{m} \mathrm{x} \Lambda \mathrm{m} \mathrm{y})=\mu_{\mathrm{B}}(\mathrm{m} \Theta(\mathrm{a}) \Lambda \mathrm{m} \Theta(\mathrm{b}))$

$=\mu_{\mathrm{B}}(\Theta(\mathrm{m} \mathrm{a}) \Lambda \theta(\mathrm{mb}))$

$=\mu_{\mathrm{B}}(\theta(\mathrm{ma} \Lambda \mathrm{mb}))$

$=\mu_{\mathrm{\theta}^{-1}(\mathrm{~B})}(\mathrm{m} \mathrm{a} \Lambda \mathrm{m} \mathrm{b})$

$\geq \min \left\{\mu_{\theta^{-1}(\mathrm{~B})}(\mathrm{ma}), \mu_{\theta^{-1}(\mathrm{~B})}(\mathrm{m} \mathrm{b})\right\}$

$\geq \min \left\{\mu_{\mathrm{B}} \Theta(\mathrm{m} \mathrm{a}), \mu_{\mathrm{B}} \Theta(\mathrm{mb})\right)$

$\geq \min \left\{\mu_{\mathrm{B}} \mathrm{m} \theta(\mathrm{a}), \mu_{\mathrm{B}} \mathrm{m} \theta(\mathrm{b})\right)$

$\geq \min \left\{\mu_{\mathrm{B}}(\mathrm{m} \mathrm{x}), \mu_{\mathrm{B}}(\mathrm{m} \mathrm{y})\right\}$

$\mathrm{B}$ is a fuzzy lattice ordered group of $\mathrm{G}$ '. 
Proposition 3.3: If $\left\{A_{i}\right\}$ is a family of fuzzy lattice ordered m-group of $G$ then $\cap A_{i}$ is a fuzzy lattice ordered m-group of $\mathrm{G}$ where $\cap \mathrm{A}_{\mathrm{i}}=\left\{\mathrm{x}, \Lambda \mu_{A i}(\mathrm{x}) / \mathrm{x} \in \mathrm{G}\right\}$

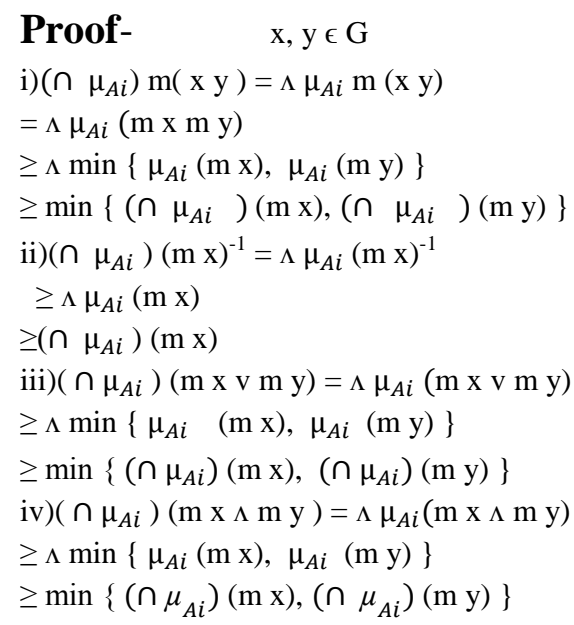

Proposition 3.4: If A is a fuzzy set in G such that all nonempty level subset

$\mathrm{U}(\mathrm{A} ; \mathrm{t})$ is a fuzzy lattice ordered $\mathrm{m}$-group of $\mathrm{G}$ then $\mathrm{A}$ is fuzzy lattice ordered $\mathrm{m}$-group of $\mathrm{G}$.

Proof- Let $x, y \in U(A ; t)$, we have $A(m x) \geq t$ and $A$ ( $m y) \geq t$. So that $A(m(x y)) \geq t$

i) $\mathrm{A}(\mathrm{m}(\mathrm{x}$ y) $) \geq \mathrm{t}$

$\geq \min \{\mathrm{t}, \mathrm{t}\}$

$\geq \min \{\mathrm{A}(\mathrm{mx}), \mathrm{A}(\mathrm{my})\}$

ii) $A\left((m x)^{-1}\right) \geq t=A(m x)$

iii) $\mathrm{A}(\mathrm{m} x \mathrm{v}$ my $) \geq \mathrm{t}$

$\geq \min \{t, t\}$

$\geq \min \{\mathrm{A}(\mathrm{mx}), \mathrm{A}(\mathrm{my})\}$

iv) $\mathrm{A}(\mathrm{m} \times \wedge \mathrm{my}) \geq \mathrm{t}$

$\geq \min \{\mathrm{t}, \mathrm{t}\}$

$\geq \min \{\mathrm{A}(\mathrm{m} \mathrm{x}), \mathrm{A}(\mathrm{my})\}$

Therefore A is a fuzzy lattice ordered m-group.

Proposition 3.5: Let A be a fuzzy lattice ordered $\mathrm{m}$ group of G. Let $A^{*}$ be a fuzzy set in $\mathrm{G}$ defined by $\mathrm{A}^{*}(\mathrm{x})=$ $\mathrm{A}(\mathrm{x})+1-\mathrm{A}(\mathrm{e})$ for all $\mathrm{x} \in \mathrm{G}$. Then $\mathrm{A}^{*}$ is a fuzzy lattice ordered m-group of $\mathrm{G}$ containing A.

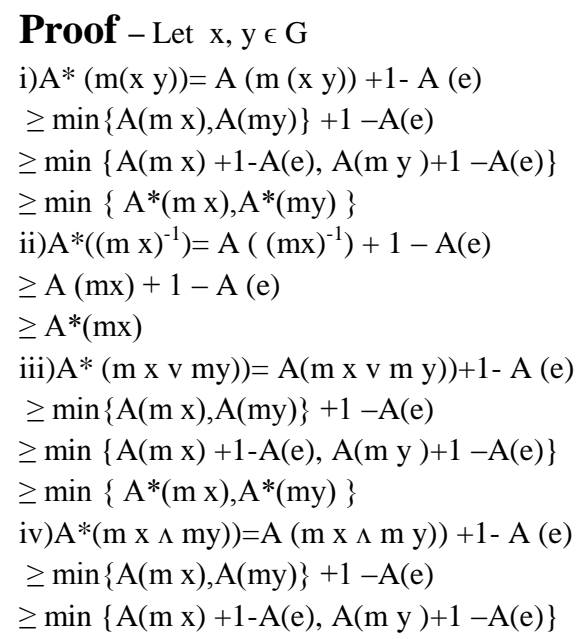

$\geq \min \left\{\mathrm{A}^{*}(\mathrm{mx}), \mathrm{A}^{*}(\mathrm{my})\right\}$

Also $\mathrm{A}(\mathrm{x}) \leq \mathrm{A}^{*}(\mathrm{x})$ for all $\mathrm{x} \in \mathrm{G}$.

Therefore $A^{*}$ is a fuzzy lattice ordered m-group of $G$ containing A.

Proposition 3.6:If A is a fuzzy lattice ordered $\mathrm{m}$ group of $\mathrm{G}$ and $\Theta$ is a m-homomorphism of $\mathrm{G}$ then the fuzzy set $A^{\theta}=\left\{\left\langle m x ; \mu_{A^{o}}(m x)\right\rangle, x \in G\right\}$ is a fuzzy lattice ordered $\mathrm{m}$-group.

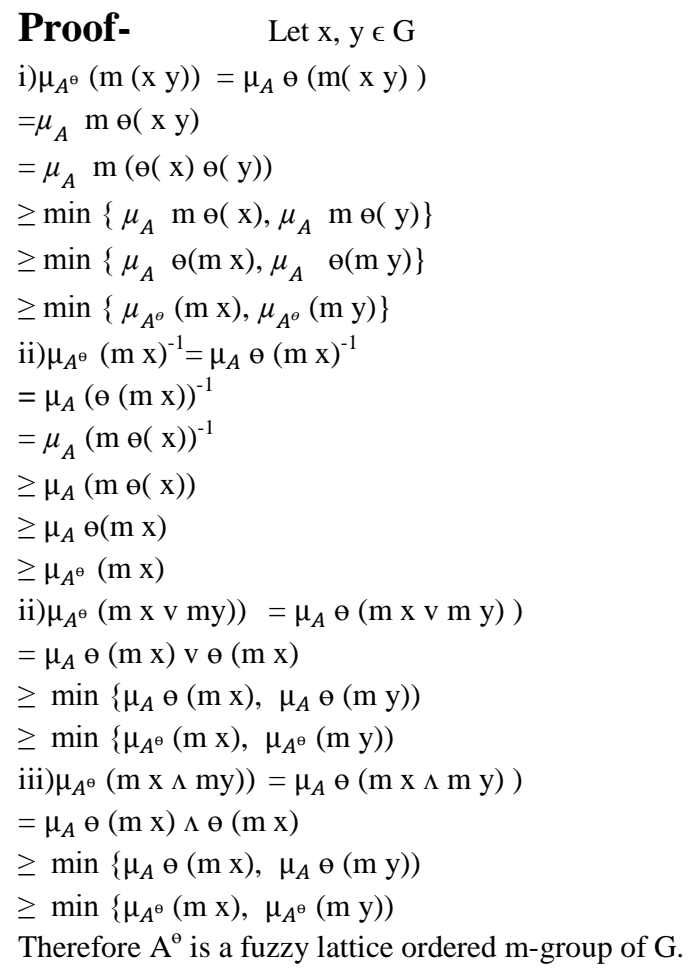

Proposition 3.7: Let $\mathrm{T}$ be a continuous t- norm and let $\mathrm{f}$ be a m-homomorphism on G. If $\mu$ is a fuzzy lattice ordered m-group on $\mathrm{G}$ then $\mu^{\mathrm{f}}$ is a fuzzy lattice ordered $\mathrm{m}$ group of $f(G)$.

Proof- Let $A_{1}=f^{-1}\left(\mathrm{~m} \mathrm{y}_{1}\right), A_{2}=f^{-1}\left(\mathrm{~m} \mathrm{y}_{2}\right), \quad A_{12}=f$ ${ }^{1}\left(\mathrm{~m}\left(\mathrm{y}_{1} \mathrm{y}_{2}\right)\right.$

Consider $A_{1} A_{2}=\left\{m x \in G / m x=m x_{1} m_{x}\right.$ for $m_{x} \in A_{1}$, $\left.\mathrm{m} \mathrm{x}_{2} \in \mathrm{A}_{2}\right\}$

If $m x \in A_{1} A_{2}$ then $m x=m_{1} m_{2}$ \&

$\mathrm{f}(\mathrm{mx})=\mathrm{f}\left(\mathrm{mx}_{1} \mathrm{mx}_{2}\right)=\mathrm{f}\left(\mathrm{mx}_{1}\right) \mathrm{f}\left(\mathrm{m} \mathrm{x}_{2}\right)$

$=\mathrm{m}_{1} \mathrm{~m}_{2} \quad=\mathrm{m}\left(\mathrm{y}_{1} \mathrm{y}_{2}\right)$

$\mathrm{mx} \in \mathrm{f}^{-1}\left(\mathrm{~m}\left(\mathrm{y}_{1} \mathrm{y}_{2}\right)\right.$ therefore $\mathrm{A}_{1} \mathrm{~A}_{2} \mathrm{C} \mathrm{A}_{12}$

i) $\mu^{\mathrm{f}}\left(\mathrm{m}\left(\mathrm{y}_{1} \mathrm{y}_{2}\right)\right)$

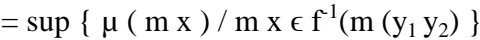

$=\sup \left\{\mu(\mathrm{mx}) / \mathrm{mx \in A} \mathrm{A}_{12}\right\}$

$\geq \sup \left\{\mu(m x) / m x \in A_{1} A_{2}\right\}$

$\geq \sup \left\{\mu\left(\mathrm{mx}_{1} \mathrm{mx}_{2}\right) / \mathrm{mx}_{1} \in \mathrm{A}_{1}, \mathrm{mx}_{2} \in \mathrm{A}_{2}\right\}$

$\geq \sup \left\{\mathrm{T}\left(\mu\left(\mathrm{mx}_{1}\right), \mathrm{T}\left(\mu\left(\mathrm{mx}_{2}\right)\right) / \mathrm{mx}_{1} \in \mathrm{A}_{1}, \mathrm{~m} \mathrm{x}_{2} \in \mathrm{A}_{2}\right\}\right.$

$\geq \mathrm{T}\left[\sup \left\{\mu\left(\mathrm{mx}_{1)} / \mathrm{mx}_{1} \in \mathrm{A}_{1}\right\}, \sup \left\{\mu\left(\mathrm{mx}_{2}\right) / \mathrm{mx}_{2} \in \mathrm{A}_{2}\right\}\right]\right.$

$\geq \mathrm{T}\left[\sup \left\{\mu\left(\mathrm{mx}_{1)} / \mathrm{m} \mathrm{x}_{1} \in \mathrm{f}^{-1}\left(\mathrm{~m} \mathrm{y}_{1}\right)\right\}, \sup \left\{\mu\left(\mathrm{m} \mathrm{x}_{2}\right) / \mathrm{m} \mathrm{x}_{2} \in \mathrm{f}\right.\right.\right.$

$\left.\left.{ }^{1}\left(\mathrm{~m} \mathrm{y}_{2}\right)\right\}\right]$

$\geq \mathrm{T}\left\{\mu^{\mathrm{f}}\left(\mathrm{m} \mathrm{y}_{1}\right), \mu^{\mathrm{f}}\left(\mathrm{m} \mathrm{y}_{2}\right)\right\}$

ii) $\mu^{\mathrm{f}}\left((\mathrm{m} \mathrm{y})^{-1}\right)$ 


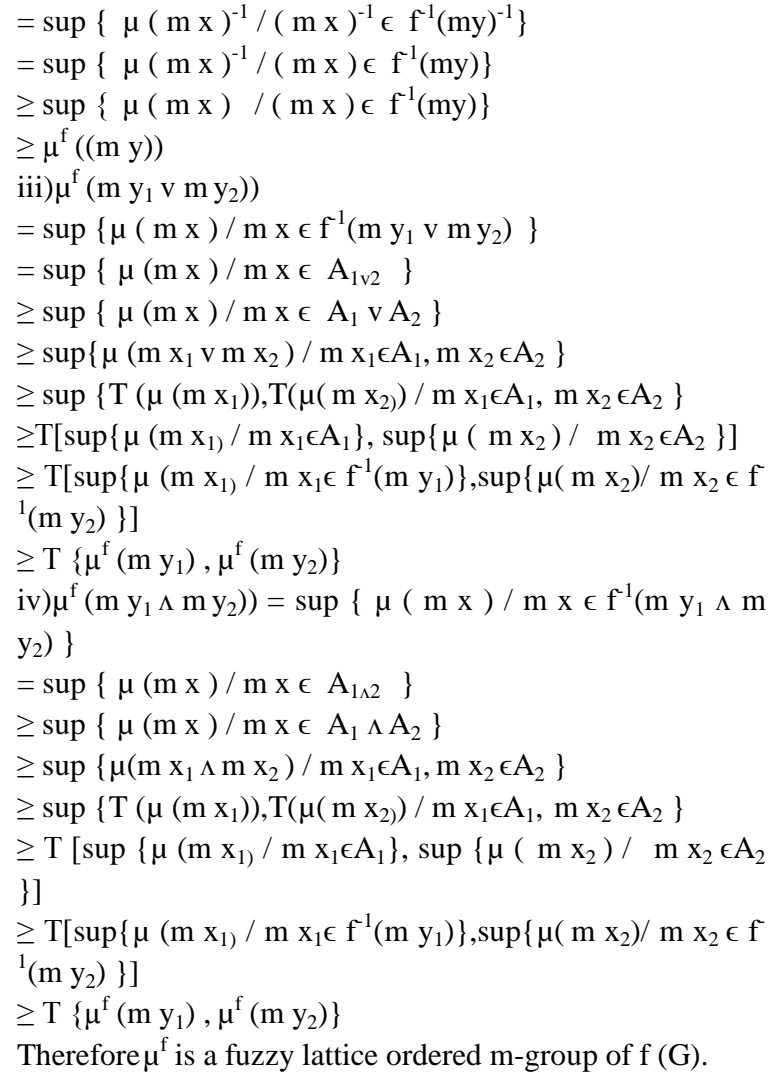

\section{Proposition 3.8:}

Let $\mathrm{T}$ be a t-norm.

Then every sensible fuzzy lattice ordered $m$ group is fuzzy lattice ordered m-group of $\mathrm{G}$.

Proof-A is sensible fuzzy lattice ordered m-group then we have

i) $A(m(x y)) \geq T[A(m x), A(m y)]$

ii) $\mathrm{A}\left((\mathrm{mx})^{-1}\right) \geq \mathrm{A}(\mathrm{mx})$

iii) $A(m x v m y) \geq T[A(m x), A(m y)]$

iv) $A(m x \wedge m y) \geq T[A(m x), A(m y)]$

i) $\min \{\mathrm{A}(\mathrm{m} x), \mathrm{A}(\mathrm{my})\}$

$=\mathrm{T}[\min \{\mathrm{A}(\mathrm{mx}), \mathrm{A}(\mathrm{my})\}, \min \{\mathrm{A}(\mathrm{mx}), \mathrm{A}(\mathrm{my})]$

$=\min \{\mathrm{T}[\mathrm{A}(\mathrm{mx}), \mathrm{A}(\mathrm{my})], \mathrm{T}[\mathrm{A}(\mathrm{mx}), \mathrm{A}(\mathrm{my})]\}$

$=\mathrm{T}[\mathrm{A}(\mathrm{m} \mathrm{x}), \mathrm{A}(\mathrm{my})]$

$\leq \mathrm{A}(\mathrm{m}(\mathrm{x} \mathrm{y}))$

ii) $\mathrm{A}\left((\mathrm{mx})^{-1}\right) \geq \mathrm{A}(\mathrm{mx})$

iii) $\min \{\mathrm{A}(\mathrm{mx}), \mathrm{A}(\mathrm{my})\}$

$=\mathrm{T}[\min \{\mathrm{A}(\mathrm{mx}), \mathrm{A}(\mathrm{my})\}, \min \{\mathrm{A}(\mathrm{mx}), \mathrm{A}(\mathrm{my})=\min \{\mathrm{T}[\mathrm{A}(\mathrm{m}$

$\mathrm{x}), \mathrm{A}(\mathrm{my})], \mathrm{T}[\mathrm{A}(\mathrm{m} \mathrm{x}), \mathrm{A}(\mathrm{my})]\}$

$=\mathrm{T}[\mathrm{A}(\mathrm{m} \mathrm{x}), \mathrm{A}(\mathrm{my})]$

$\leq \mathrm{A}(\mathrm{mx} \vee \mathrm{m} \mathrm{y}))$

iv)min $\{\mathrm{A}(\mathrm{m} x), \mathrm{A}(\mathrm{my})\}$

$=\mathrm{T}[\min \{\mathrm{A}(\mathrm{mx}), \mathrm{A}(\mathrm{my})\}, \min \{\mathrm{A}(\mathrm{mx}), \mathrm{A}(\mathrm{my})\}]=\min \{\mathrm{T}[\mathrm{A}(\mathrm{m}$

$\mathrm{x}), \mathrm{A}(\mathrm{my})], \mathrm{T}[\mathrm{A}(\mathrm{mx}), \mathrm{A}(\mathrm{my})]\}$

$=\mathrm{T}[\mathrm{A}(\mathrm{mx}), \mathrm{A}(\mathrm{my})]$

$\leq \mathrm{A}(\mathrm{mx} \wedge \mathrm{m} \mathrm{y}))$

Therefore A is a fuzzy lattice ordered m-group of G.

\section{Proposition3.9:}

An onto m-homomorphic image of fuzzy lattice ordered mgroup with sup property is a fuzzy lattice ordered m-group.

Proof: Let $f: G \rightarrow G$ ' be a onto

m-homomorphism of $\mathrm{G}$ and let A be a fuzzy

lattice ordered $\mathrm{m}$-group of $\mathrm{G}$ with sup

property.

Let $m x^{\prime}, m^{\prime} \in G^{\prime}$

Let $\mathrm{mx}_{0} \in \mathrm{f}^{-1}(\mathrm{~m} \mathrm{x}), \mathrm{my}_{0} \in \mathrm{f}^{-1}\left(\mathrm{~m} \mathrm{y}^{\prime}\right)$

be such that

$\mathrm{A}\left(\mathrm{mx}_{0}\right)=\sup \left\{\mathrm{A}(\mathrm{mx}) / \mathrm{mx} \in \mathrm{f}^{-1}\left(\mathrm{mx}^{\prime}\right)\right\} \&$

$\mathrm{A}\left(\mathrm{m} \mathrm{y}_{0}\right)=\sup \left\{\mathrm{A}(\mathrm{m} \mathrm{y}) / \mathrm{m} \mathrm{y} \in \mathrm{f}^{-1}\left(\mathrm{~m} \mathrm{y}^{\prime}\right)\right\}$

i) $A^{f}\left(m\left(x^{\prime} y^{\prime}\right)\right)=\sup \left\{A(z) / z \in f^{-1}\left(m\left(x^{\prime} y^{\prime}\right)\right)\right\}$

$\left.=\sup \left\{\mathrm{A}(\mathrm{z}) / \mathrm{z} \in \mathrm{f}^{-1}\left(\mathrm{~m} \mathrm{x}^{\prime} \mathrm{my}\right)\right)\right\}$

$\geq \sup \left\{\mathrm{A}\left(\mathrm{mx}_{0} \mathrm{~m} \mathrm{y}_{0}\right) / \mathrm{mx}_{0} \in \mathrm{f}^{-1}\left(\mathrm{mx}^{\prime}\right), \mathrm{my}_{0} \in \mathrm{f}^{-1}\left(\mathrm{~m} \mathrm{y}^{\prime}\right)\right\}$

$\geq \sup \left\{\mathrm{A}\left(\mathrm{mx}_{0} \mathrm{y}_{0}\right) / \mathrm{mx}_{0} \in \mathrm{f}^{-1}\left(\mathrm{mx}^{\prime}\right), \mathrm{my}_{0} \in \mathrm{f}^{-1}\left(\mathrm{~m} \mathrm{y}^{\prime}\right)\right\}$

$\geq \sup \left\{\min \left\{\mathrm{A}\left(\mathrm{mx}_{0}\right), \mathrm{A}\left(\mathrm{m} \mathrm{y}_{0}\right)\right) / \mathrm{mx}_{0} \epsilon\right.$

$\left.\mathrm{f}^{-1}\left(\mathrm{~m} \mathrm{x}^{\prime}\right), \mathrm{my}_{0} \in \mathrm{f}^{-1}\left(\mathrm{~m} \mathrm{y}^{\prime}\right)\right\}$

$\geq \min \left\{\sup \left\{\mathrm{A}\left(\mathrm{mx}_{0}\right) / \mathrm{mx}_{0} \epsilon\right.\right.$

$\left.\mathrm{f}^{-1}\left(\mathrm{~m} \mathrm{x}^{\prime}\right)\right\}, \sup \left\{\mathrm{A}\left(\mathrm{my}_{0}\right) / \mathrm{my}_{0} \in \mathrm{f}^{-1}\left(\mathrm{~m} \mathrm{y}^{\prime}\right)\right.$

$\geq \min \left\{A^{\mathrm{f}}\left(m x^{\prime}\right), A^{\mathrm{f}}\left(m x^{\prime}\right)\right\}$

ii) $A^{\mathrm{f}}\left(\left(\mathrm{mx} \mathrm{x}^{\prime}\right)^{-1}\right)=\sup \left\{\mathrm{A}\left(\mathrm{mx}_{0}\right)^{-1} /\left(\mathrm{mx}_{0}\right)^{-1} \in \mathrm{f}^{-1}\left(\mathrm{mx}^{\prime}\right)^{-1}\right\}$

$=\sup \left\{\mathrm{A}\left(\mathrm{mx}_{0}\right)^{-1} /\left(\mathrm{mx}_{0}\right) \in \mathrm{f}^{-1}\left(\mathrm{mx}^{\prime}\right)\right\}$

$\geq \sup \left\{\mathrm{A}\left(\mathrm{mx}_{0}\right) /\left(\mathrm{mx}_{0}\right) \in \mathrm{f}^{-1}\left(\mathrm{mx}^{\prime}\right)\right\}$

$\geq \mathrm{A}^{\mathrm{f}}((\mathrm{m} \mathrm{x}))$

iii) $\mathrm{A}^{\mathrm{f}}\left(\mathrm{m} \mathrm{x}^{\prime} \mathrm{v}\right.$ my' $\left.)\right)=\sup \{\mathrm{A}(\mathrm{z}) /$

$\left.\left.\mathrm{z} \in \mathrm{f}^{-1}\left(\mathrm{~m} \mathrm{x}^{\prime} \mathrm{v} \mathrm{m} \mathrm{y}^{\prime}\right)\right)\right\}$

$\left.\left.\geq \sup \left\{\mathrm{A}(\mathrm{z}) / \mathrm{z} \in \mathrm{f}^{-1}\left(\mathrm{mx}^{\prime}\right) \mathrm{vf}^{1} \mathrm{~m} \mathrm{y}^{\prime}\right)\right)\right\}$

$\geq \sup \left\{\mathrm{A}\left(\mathrm{mx}_{0} \vee \mathrm{m} \mathrm{y}_{0}\right) / \mathrm{mx}_{0} \in \mathrm{f}^{-1}\left(\mathrm{mx}^{\prime}\right), \mathrm{my}_{0} \in \mathrm{f}^{-1}\left(\mathrm{~m} \mathrm{y}^{\prime}\right)\right\}$

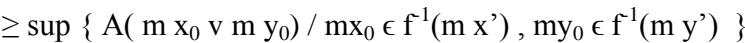

$\geq \sup \left\{\min \left\{\mathrm{A}\left(\mathrm{mx}_{0}\right), \mathrm{A}\left(\mathrm{m} \mathrm{y}_{0}\right)\right) / \mathrm{mx}_{0}\right.$

$\left.\in \mathrm{f}^{-1}(\mathrm{~m} \mathrm{x}), \mathrm{my}_{0} \in \mathrm{f}^{-1}\left(\mathrm{~m} \mathrm{y}^{\prime}\right)\right\}$

$\geq \min \left\{\sup \left\{\mathrm{A}\left(\mathrm{mx}_{0}\right) / \mathrm{mx}_{0} \epsilon\right.\right.$

$\left.\mathrm{f}^{-1}\left(\mathrm{~m} \mathrm{x}^{\prime}\right)\right\}, \sup \left\{\mathrm{A}\left(\mathrm{my}_{0}\right) / \mathrm{my}_{0} \in \mathrm{f}^{-1}\left(\mathrm{~m} \mathrm{y}^{\prime}\right)\right.$

$\geq \min \left\{\mathrm{A}^{\mathrm{f}}\left(\mathrm{m} \mathrm{x}^{\prime}\right), \mathrm{A}^{\mathrm{f}}\left(\mathrm{m} \mathrm{x}^{\prime}\right)\right\}$

iv) $A^{f}\left(m x^{\prime} \Lambda\right.$ my') $)=\sup \{A(z) / z \epsilon$

$\left.\left.\mathrm{f}^{-1}\left(\mathrm{~m} \mathrm{x} \mathrm{x}^{\prime} \Lambda \mathrm{m} \mathrm{y}^{\prime}\right)\right)\right\}$

$\left.\left.\geq \sup \left\{\mathrm{A}(\mathrm{z}) / \mathrm{z} \in \mathrm{f}^{-1}\left(\mathrm{mx}^{\prime}\right) \Lambda \mathrm{f}^{-1} \mathrm{~m} \mathrm{y}^{\prime}\right)\right)\right\}$

$\geq \sup \left\{\mathrm{A}\left(\mathrm{mx}_{0} \wedge \mathrm{m}_{0}\right) / \mathrm{mx}_{0} \in \mathrm{f}^{-1}\left(\mathrm{mx}^{\prime}\right), \mathrm{my}_{0} \in \mathrm{f}^{-1}\left(\mathrm{~m} \mathrm{y}^{\prime}\right)\right\}$

$\geq \sup \left\{\mathrm{A}\left(\mathrm{mx}_{0} \Lambda \mathrm{m} \mathrm{y}_{0}\right) / \mathrm{mx}_{0} \in \mathrm{f}^{-1}\left(\mathrm{mx}^{\prime}\right), \mathrm{my}_{0} \in \mathrm{f}^{-1}\left(\mathrm{~m} \mathrm{y}^{\prime}\right)\right\}$

$\geq \sup \left\{\min \left\{\mathrm{A}\left(\mathrm{mx}_{0}\right), \mathrm{A}\left(\mathrm{m}_{0}\right)\right) / \mathrm{mx}_{0}\right.$

$\left.\epsilon \mathrm{f}^{-1}\left(\mathrm{mx} \mathrm{x}^{\prime}\right), \mathrm{my}_{0} \in \mathrm{f}^{-1}\left(\mathrm{~m} \mathrm{y}^{\prime}\right)\right\}$

$\geq \min \left\{\sup \left\{\mathrm{A}\left(\mathrm{mx}_{0}\right) / \mathrm{mx}_{0} \epsilon\right.\right.$

$\left.\mathrm{f}^{-1}\left(\mathrm{~m} \mathrm{x}^{\prime}\right)\right\}, \sup \left\{\mathrm{A}\left(\mathrm{my}_{0}\right) / \mathrm{my}_{0} \in \mathrm{f}^{-1}\left(\mathrm{~m} \mathrm{y}^{\prime}\right)\right.$

$\geq \min \left\{A^{f}\left(m x^{\prime}\right), A^{f}\left(m x^{\prime}\right)\right\}$

Proposition 3.10: Let $f: G \rightarrow G$ ' be a lattice group $m$ homomorphism and A be a fuzzy lattice ordered m-group of $G^{\prime}$ then $f^{1}(A)$ is a fuzzy lattice ordered m-group of $G$.

Proof -Let $m x, m$ y $\in G$ and $A$ be a fuzzy lattice ordered m-group of G'.

i) $f^{-1}(A)(m(x y))=\operatorname{A~f}(m(x y))$

$=A(f(m x) f(m y))$

$=A(\mathrm{~m} \mathrm{f}(\mathrm{x}) \mathrm{mf}(\mathrm{y}))$

$=A(m f(x) f(y))$

$\geq \min \{\mathrm{A}(\operatorname{mf}(\mathrm{x})), \mathrm{A}(\operatorname{mf}(\mathrm{y}))\}$

$\geq \min \{\mathrm{A}(\mathrm{f}(\mathrm{m} x)), \mathrm{A}(\mathrm{f}(\mathrm{my}))\}$ 
$\geq \min \left\{\mathrm{f}^{-1}(\mathrm{~A})(\mathrm{mx}), \mathrm{f}^{-1}(\mathrm{~A})(\mathrm{mx})\right\}$

i) $\mathrm{f}^{-1}(\mathrm{~A})\left((\mathrm{mx})^{-1}\right) \quad=\operatorname{Af}\left((\mathrm{mx})^{-1}\right)$

$=A(f(m x))^{-1}$

$=\mathrm{A}(\mathrm{m} \mathrm{f}(\mathrm{x}))^{-1}$

$\geq \mathrm{A}(\mathrm{m} \mathrm{f}(\mathrm{x}))$

$\geq \mathrm{A}(\mathrm{f}(\mathrm{mx}))$

$\geq \mathrm{f}^{-1}(\mathrm{~A})(\mathrm{mx})$

ii) $\left.\left.f^{-1}(A)(m x \vee m y)\right)=\operatorname{Af}(m x \vee m y)\right)$

$=A(f(m x) \vee f(m y))$

$=A(m f(x) \vee \operatorname{m~f}(y))$

$\geq \min \{\mathrm{A}(\operatorname{mf}(\mathrm{x})), \mathrm{A}(\operatorname{mf}(\mathrm{y}))\}$

$\geq \min \{\mathrm{A}(\mathrm{f}(\mathrm{mx})), \mathrm{A}(\mathrm{f}(\mathrm{my}))\}$

$\geq \min \left\{\mathrm{f}^{-1}(\mathrm{~A})(\mathrm{mx}), \mathrm{f}^{-1}(\mathrm{~A})(\mathrm{mx})\right\}$

iii) $\left.\left.f^{-1}(\mathrm{~A})(\mathrm{mx} \wedge \mathrm{m} \mathrm{y})\right)=\mathrm{A} \mathrm{f}(\mathrm{mx} \wedge \mathrm{m} \mathrm{y})\right)$

$=A(f(m x) \wedge f(m y))$

$=\mathrm{A}(\mathrm{mf}(\mathrm{x}) \Lambda \mathrm{mf}(\mathrm{y}))$

$\geq \min \{\mathrm{A}(\operatorname{mf}(\mathrm{x})), \mathrm{A}(\operatorname{mf}(\mathrm{y}))\}$

$\geq \min \{\mathrm{A}(\mathrm{f}(\mathrm{m} \mathrm{x})), \mathrm{A}(\mathrm{f}(\mathrm{my}))\}$

$\geq \min \left\{\mathrm{f}^{-1}(\mathrm{~A})(\mathrm{mx}), \mathrm{f}^{-1}(\mathrm{~A})(\mathrm{mx})\right\}$

Therefore $\mathrm{f}^{-1}(\mathrm{~A})$ is a fuzzy lattice ordered $\mathrm{m}$-group of $\mathrm{G}$.

\section{DIRECT PRODUCT OF FUZZY LATTICE ORDERED M-GROUP}

Definition: 4.1 Let $A_{i}$ be a fuzzy lattice ordered mgroup of $G_{i}$, for $i=1,2, \ldots \ldots, n$. Then the product $A_{i}(i=$ $1,2, \ldots \ldots n)$ is the function $A_{1} x A_{2} x \ldots \ldots . . . \times A_{n}: G_{1} x G_{2} x$ $\ldots \ldots . . \times G_{n} \rightarrow L$ defined by $\left(A_{1} x A_{2} x \ldots \ldots . . . \times A_{n}\right) m(x$

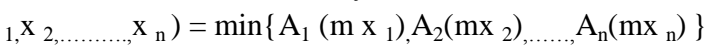

Proposition 4.2: The direct product of fuzzy lattice ordered $\mathrm{m}$ groups is a fuzzy lattice ordered $\mathrm{m}$-group.

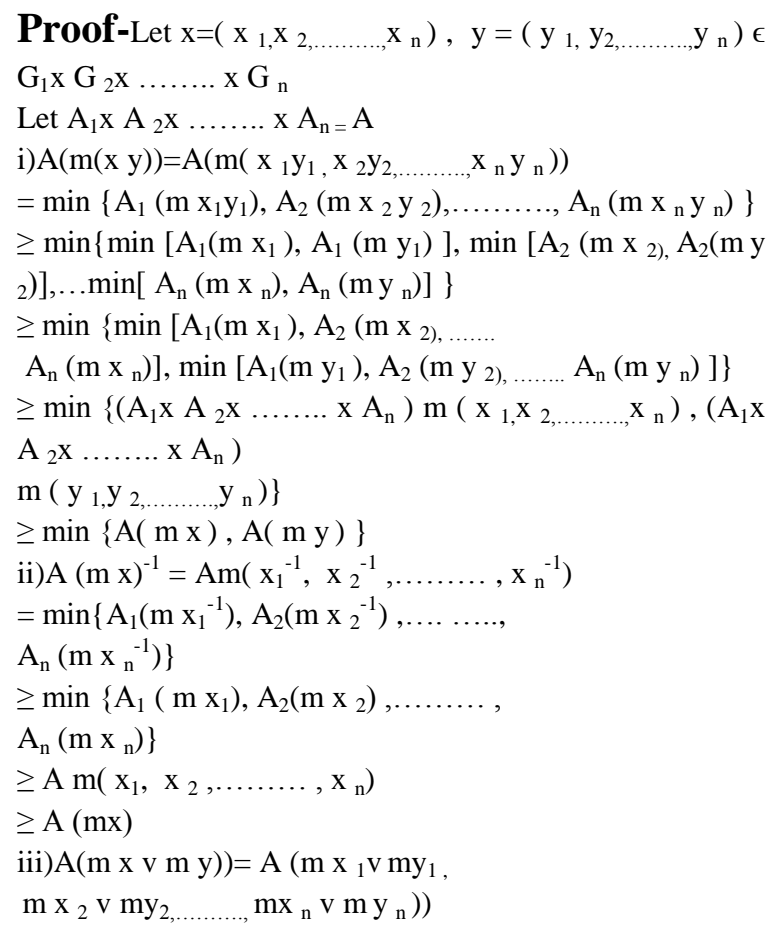

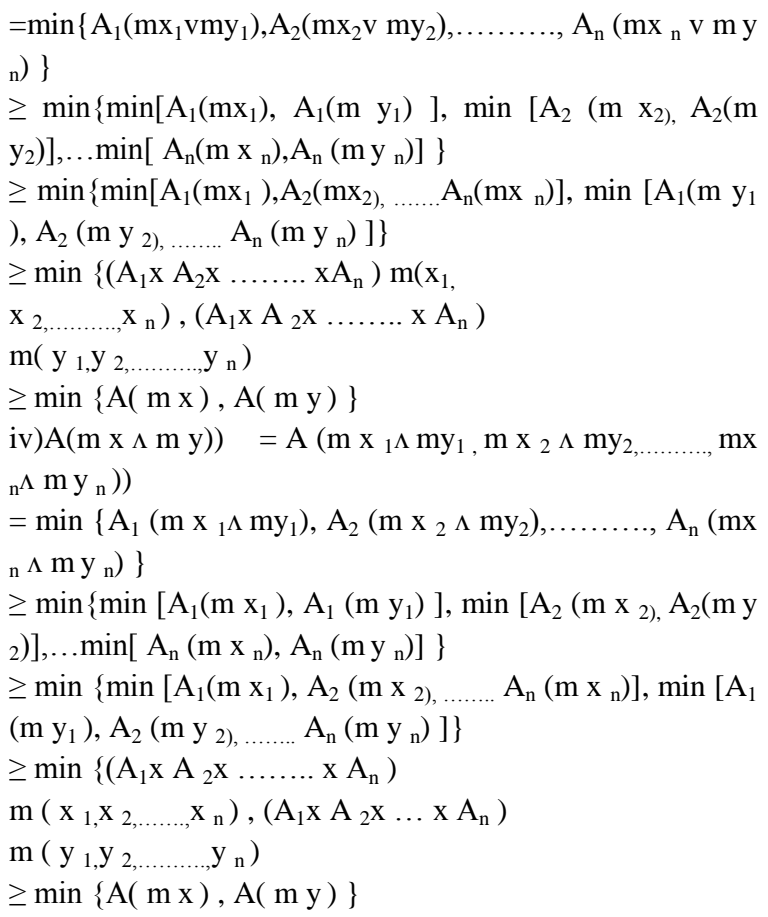

\section{CONCLUSION}

In this paper we studied the notion of fuzzy lattice ordered $\mathrm{m}$-groups and investigated some of its basic properties. We also studied the homomorphic image, pre-image of fuzzy lattice ordered m-groups, arbitrary family of fuzzy lattice ordered m-groups and fuzzy lattice ordered mgroups using $\mathrm{T}$-norms

Applications: Lattice structure has been found to be extremely important in the areas of quantum logic, Erogodic theory, Reynold's operations, Soft Computing, Communication system, Information analysis system, artificial intelligences and physical sciences.

\section{ACKNOWLEDGEMENT}

The authors is highly grateful to the referees for their valuable comments and suggestions for improving the paper.

\section{REFERENCES}

[1] Ajmal N and K.V.Thomas, The Lattice of Fuzzy subgroups and fuzzy normal subgroups, Inform. sci. 76 (1994), 1 - 11 .

[2] Birkoff, G : Lattice theory 3rd edn. Amer.Math. Soc. Colloquium pub.25 (1984).

[3] G.S.V. Satya Saibaba. Fuzzy lattice ordered groups, South east Asian Bulletin of Mathematics 32,749-766 (2008).

[4] J.A. Goguen : L - Fuzzy Sets, J. Math Anal.Appl. 18, 145-174 (1967) 
[5] L. A.Zadeh : Fuzzy sets, Inform and Control, 8, 338353 (1965).

[6] M.Marudai \& V. Rajendran: Characterization of Fuzzy Lattices on a Group International Journal of Computer Applications with Respect to T-Norms, 8(8),0975 - 8887 ( 2010)

[7] Mordeson and D.S. Malik : Fuzzy Commutative Algebra, World Scientific Publishing Co. Pvt. Ltd.

[8] Nanda, S : Fuzzy Lattices, Bulletin Calcutta Math. Soc. 81 (1989) $1-2$.
[9] Rosenfeld : Fuzzy groups, J. Math. Anal. Appl. 35, $512-517$ (1971)

[10] S. Subramanian, R Nagrajan \& Chellappa, Structure Properties of M-Fuzzy Groups Applied Mathematical Sciences,6(11),545-552( 2012)

[11] Solairaju and R. Nagarajan : Lattice Valued Q-fuzzy left R - Submodules of Neat Rings with respect to TNorms, Advances in fuzzy mathematics 4(2), 137 145 (2009).

[12] W.X.Gu. S.Y.Li and D.G.Chen, fuzzy groups with operators, fuzzy sets and system,66 (1994) ,363-371. 